\title{
Modified Muscle Transposition Procedure for a Case of Inferior Rectus Muscle Aplasia
}

\author{
Shinji Makino Kozue Hozawa Reiko Kondo Mika Kanai Haruko Suto \\ Go Mawatari Kanako Ito \\ Department of Ophthalmology, Jichi Medical University, Shimotsuke, Japan
}

\section{Key Words}

Aplasia of the inferior rectus muscle $\cdot$ Muscle transposition procedure $\cdot$ Magnetic resonance imaging

\begin{abstract}
Purpose: We report a case of inferior rectus muscle aplasia in a 65-year-old woman. Methods: Images were obtained using ocular motility photography and magnetic resonance imaging (MRI), and operative findings were analyzed. Results: A 65-year-old woman presented with marked right hypertropia. The right eye also had microcornea, iris coloboma, and completely restricted downward movement. MRI showed absence of the inferior rectus muscle in both eyes. During surgery, it was confirmed that the right inferior rectus muscle was absent. The patient underwent a muscle transposition procedure without tenotomy or muscle splitting. A superior rectus muscle tenotomy was also performed. Postoperatively, the right eye showed hypotropia and improved downward movement. Conclusion: Muscle transposition without tenotomy or tendon splitting is a surgical option for the unusual abnormality presented in this paper.

(c) 2015 S. Karger AG, Basel
\end{abstract}

\section{Introduction}

Congenital absence or aplasia of extraocular muscles is a rare cause of restrictive or paralytic strabismus [1-3]. Absence of the superior oblique muscle is the most frequently encountered abnormality, followed by absence of the inferior rectus muscle [1-3]. Generally, the standard surgical procedure is recession or tenotomy of the superior rectus muscle combined with muscle transposition for inferior rectus muscle aplasia [1-3]. In 2005, Nishida et al. [4] proposed a simpler and more effective muscle transposition procedure in

KARGER 125:s $\quad \begin{aligned} & \text { Shinji Makino } \\ & \text { Department of Ophthalmology, Jichi Medical University } \\ & \text { 3311-1 Yakushiji } \\ & \text { Shimotsuke, Tochigi 329-0498 (Japan) } \\ & \text { E-Mail makichan@jichi.ac.jp }\end{aligned}$


Makino et al.: Modified Muscle Transposition Procedure for a Case of Inferior Rectus Muscle Aplasia

which the superior and inferior rectus muscles are transposed without tenotomy or muscle splitting for abducent palsy. In this study, we present a case of aplasia of the inferior rectus muscle treated with the modified muscle transposition procedure without tenotomy or muscle splitting, as described by Nishida et al. [4].

\section{Case Report}

A 65-year-old woman was referred to Jichi Medical University Hospital because of recent deterioration of right hypertropia, which had been present since birth. The patient had no specific medical history. Best-corrected visual acuity was 0.02 and 0.01 in her right and left eye, respectively. The right eye showed marked hypertropia and completely restricted downward movement (fig. 1). Slit-lamp examination showed cortical opacities in both lenses. Microcornea and iris coloboma were also present in both eyes (fig. 2). Coronal T1weighted magnetic resonance imaging (MRI) revealed the absence of inferior rectus muscles in both eyes (fig. 3a), which was also confirmed by sagittal T1-weighted MRI (fig. 3b, c). Furthermore, the optic nerve was displaced inferiorly (arrows in fig. 3a-c); therefore, we diagnosed the marked hypertropia as aplasia of the inferior rectus muscle.

During surgery, forced duction test showed no depression below the midline in the right eye, indicating contracture of the right superior rectus muscle. Moreover, it was confirmed that the right inferior rectus muscle was absent (fig. 4). The patient underwent a muscle transposition procedure without tenotomy or muscle splitting, as described by Nishida et al. [4]. A superior rectus muscle tenotomy was also performed (fig. 5). In accordance with the procedure by Nishida et al. [4], after the horizontal recti were explored, 5-0 polyester sutures were passed through the inferior margins at a distance of $8 \mathrm{~mm}$ behind their insertion points. The same suture was passed through each scleral wall at a distance of $10 \mathrm{~mm}$ behind the naso- and temporo-inferior limbus. Then, the inferior margin of each horizontal rectus muscle was transposed naso- or temporo-inferiorly and anchored onto the sclera. Regrettably, however, surgical correction was not effective; therefore, we retransposed each horizontal rectus to the putative inferior rectus muscle insertion (fig. 5; right panel; dashed line) 7 $\mathrm{mm}$ from the limbus (fig. 5; left panel; arrows). As a result of this retransposition, ocular alignment markedly improved at the end of surgery. Postoperatively, the right eye showed hypotropia and improved downward movement (fig. 6). The patient was satisfied with the surgical outcome. No postoperative anterior segment ischemia was detected. The reported findings did not change at any point during the 1-year follow-up period.

\section{Discussion}

The reported structural anomalies of the eye globe of patients with inferior rectus muscle aplasia include microphthalmos, microcornea, coloboma, and Axenfeld-Rieger syndrome $[1-3,5]$.

Generally, the standard surgical procedure for inferior rectus muscle aplasia involves recession or tenotomy of the superior rectus muscle combined with muscle transposition $[1-3,5]$. Muscle transposition can be performed in two ways: inferior transposition of the entire muscle tendons of the medial and lateral rectus muscles (a modification of the Knapp procedure), or transposition of half or two thirds of the tendons of the medial and lateral rectus muscles (a modification of the Hummelsheim procedure) to the putative location of the missing inferior rectus muscle insertion [3]. 
Makino et al.: Modified Muscle Transposition Procedure for a Case of Inferior Rectus Muscle Aplasia

In 2003, Nishida et al. [6] reported a new muscle transposition procedure in which split temporal halves of the superior and inferior recti are transposed without tenotomy or lateral rectus muscle treatment. In order to further reduce operative damage, they developed a second new procedure that did not involve tenotomy or muscle splitting [4].

We have previously reported a patient with inferior rectus muscle aplasia who underwent a muscle transposition procedure without tenotomy or muscle splitting [7]. In this previous case, each horizontal rectus muscle was transposed onto the scleral wall at a distance of $7 \mathrm{~mm}$ behind the naso- and temporo-inferior limbus with superior rectus muscle tenotomy. The present case is the second reported case of inferior rectus muscle aplasia that underwent the above-mentioned modified procedure introduced by Nishida et al. [4]. In addition, in the present case, we modified the procedure so that each horizontal rectus muscle was transposed to nearly the putative location of the inferior rectus muscle insertion point.

The muscle transposition procedure without tenotomy or tendon splitting is a surgical option for the unusual abnormality reported in this case. Although the current findings are based on a single case, we speculate that muscle transposition to the nearly putative inferior rectus muscle insertion point as well as the use of the Hummelsheim procedure are more effective in patients with inferior rectus muscle aplasia. Long-term follow-up and additional cases are necessary to further evaluate the utility of this procedure.

\section{Disclosure Statement}

The authors have no conflicts of interest to disclose.

\section{References}

-1 Taylor RH, Kraft SP: Aplasia of the inferior rectus muscle. A case report and review of the literature. Ophthalmology 1997;104:415-418.

-2 Astle WF, Hill VE, Ells AL, Chi NTT, Martinovic E: Congenital absence of the inferior rectus muscle diagnosis and management. J AAPOS 2003;7:339-344.

-3 Matsuo T, Watanabe T, Furuse T, Hasebe S, Ohtsuki H: Case report and literature review of inferior rectus muscle aplasia in 16 Japanese patients. Strabismus 2009;17:66-74.

-4 Nishida Y, Hayashi O, Oda S, Kakinoki M, Miyake T, Inoki Y, Iwami T, Mekada A, Okada A: A simple muscle transposition procedure for abducens palsy without tenotomy or splitting muscles. Jpn J Ophthalmol 2005;49:179-180.

5 Bhate M, Martin FJ: Unilateral inferior rectus hypoplasia in a child with Axenfeld-Rieger syndrome. J AAPOS 2012;16:304-306.

-6 Nishida Y, Inatomi A, Aoki Y, Hayashi O, Iwami T, Oda S, Nakamura J, Kani K: A muscle transposition procedure for abducens palsy, in which the halves of the vertical rectus muscle bellies are sutured onto the sclera. Jpn J Ophthalmol 2003;47:281-286.

7 Makino S, Hozawa K, Kondo R, Kumagai T, Ito H, Hirabayashi R, Kanai M: Two cases of hypoplasia of the inferior rectus muscle (in Japanese). Jichi Med Univ J 2010;33:123-128. 
Case Reports in

Ophthalmology
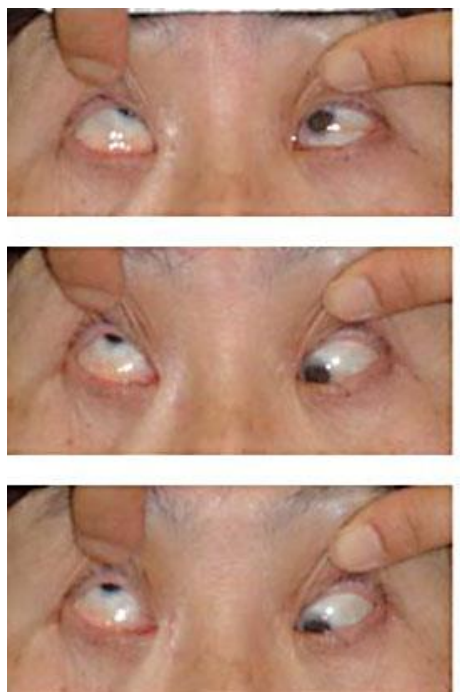

\begin{tabular}{l|l}
\hline \multicolumn{2}{l|}{ Case Rep Ophthalmol 2015;6:1-6 } \\
\hline DOI: $10.1159 / 000371508$ & $\begin{array}{l}\text { C } 2015 \text { S. Karger AG, Basel } \\
\text { www.karger.com/cop }\end{array}$ \\
\hline
\end{tabular}

Makino et al.: Modified Muscle Transposition Procedure for a Case of Inferior Rectus Muscle Aplasia
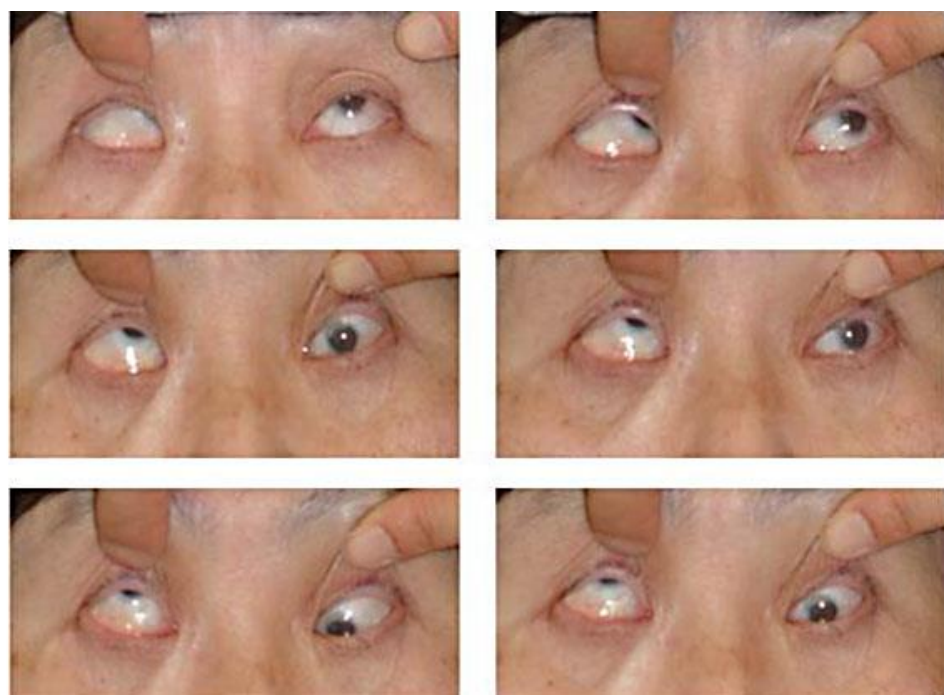

Fig. 1. Preoperative ocular motility photographs of the patient in the nine gaze positions. The right eye revealed marked hypertropia and completely restricted downward movement.
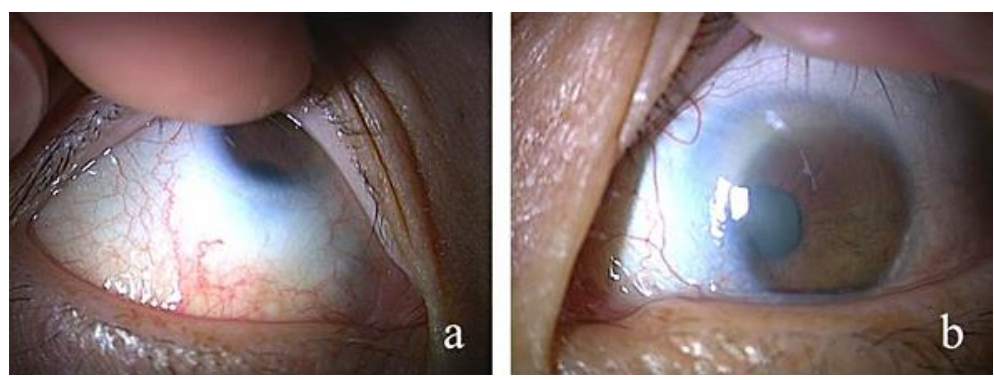

Fig. 2. Anterior segment photographs of the right (a) and left eye (b). Microcornea and iris coloboma were present in both eyes. 


\section{Case Reports in \\ Ophthalmology}

\begin{tabular}{l|l}
\hline \multicolumn{2}{l}{ Case Rep Ophthalmol 2015;6:1-6 } \\
\hline DOI: 10.1159/000371508 & $\begin{array}{l}\text { ○ 2015 S. Karger AG, Basel } \\
\text { www.karger.com/cop }\end{array}$ \\
\hline
\end{tabular}

Makino et al.: Modified Muscle Transposition Procedure for a Case of Inferior Rectus Muscle Aplasia
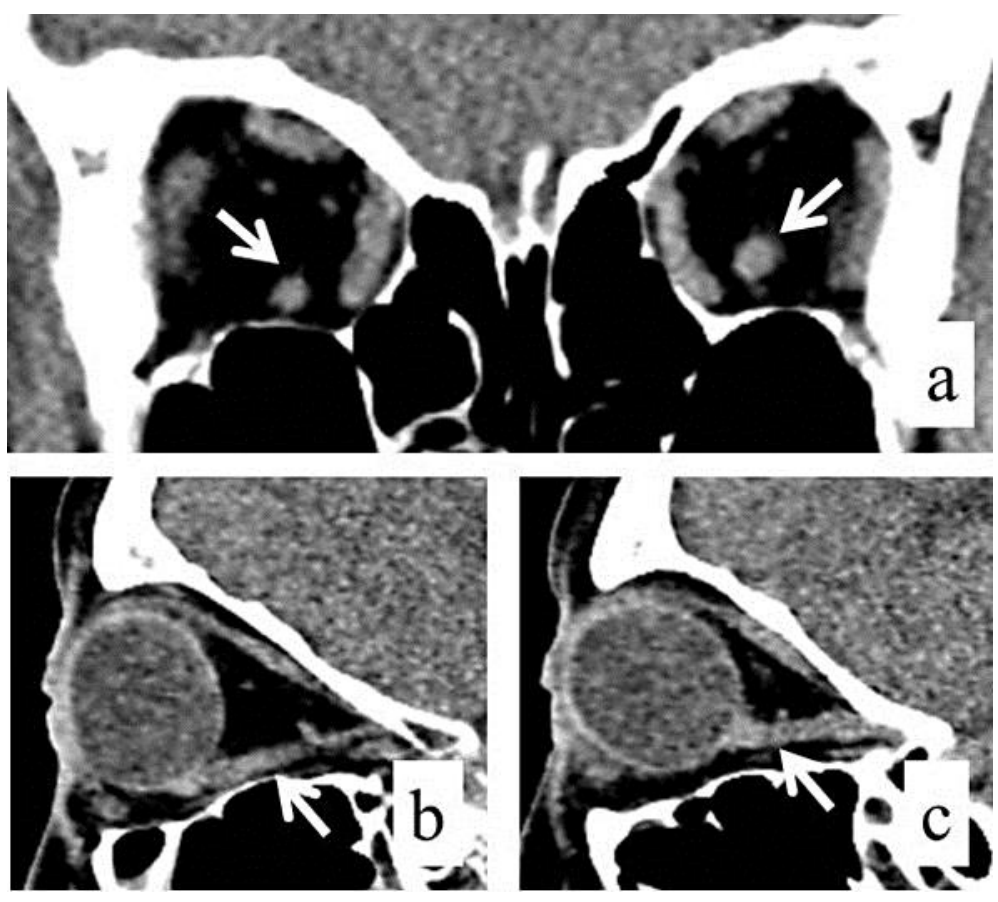

Fig. 3. a Coronal T1-weighted MRI revealed the absence of the inferior rectus muscle in both eyes. Sagittal T1-weighted MRI revealed the absence of the inferior rectus muscle in the right (b) and left (c) eye. The optic nerve was also displaced inferiorly (arrows in a-c).

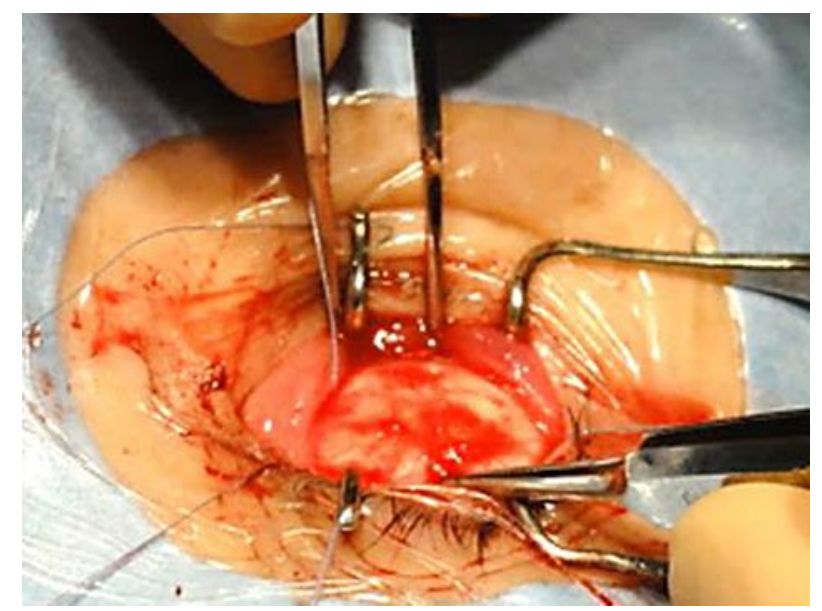

Fig. 4. Operative findings. The right inferior rectus muscle was confirmed to be absent. 
Makino et al.: Modified Muscle Transposition Procedure for a Case of Inferior Rectus Muscle Aplasia
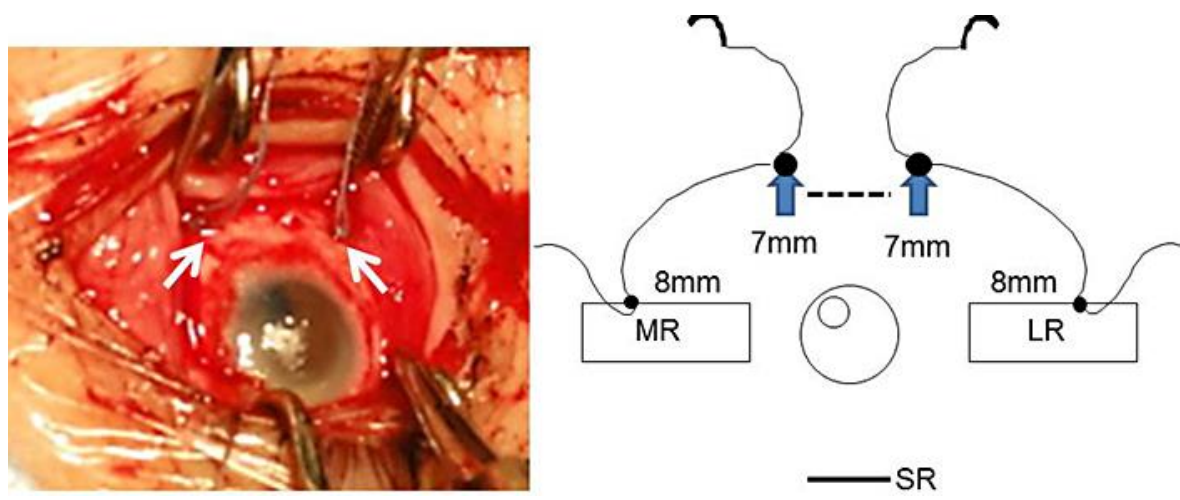

Fig. 5. Operative finding (left panel) and schematic interpretation of the muscle transposition procedure (right panel). The sutures were passed through the inferior margins at a distance of $8 \mathrm{~mm}$ behind each horizontal rectus muscle insertion. Each horizontal rectus muscle was transposed to the putative inferior rectus muscle insertion point (right panel; dashed line) $7 \mathrm{~mm}$ from the limbus and anchored onto the sclera (left panel; arrows). Abbreviations: $M R=$ medial rectus muscle; $L R=$ lateral rectus muscle; $\mathrm{SR}=$ superior rectus muscle.
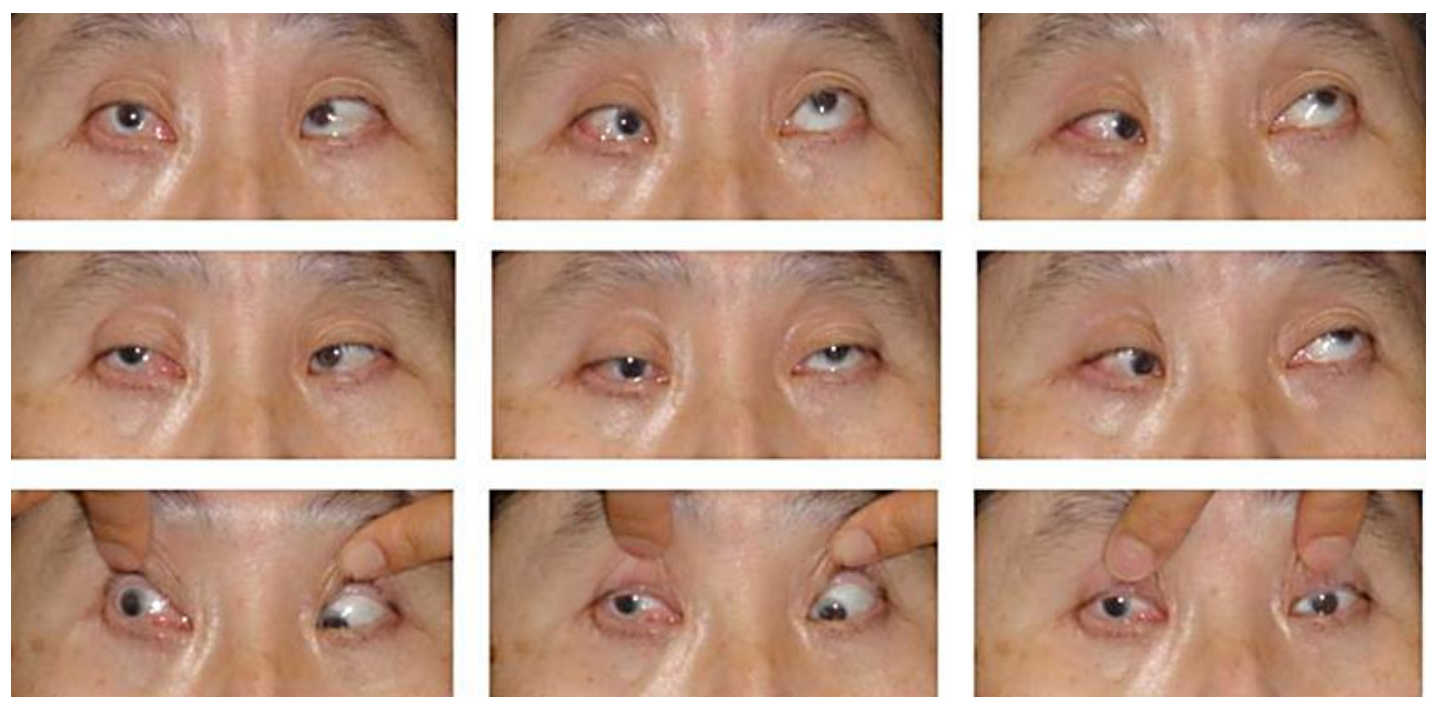

Fig. 6. Postoperative ocular motility photographs of the patient in the nine gaze positions. The right eye revealed hypotropia and improved downward movement. 